\begin{tabular}{|r|l|}
\hline \multicolumn{2}{|c|}{ Statistica Sinica Preprint No: SS-2020-0174 } \\
\hline Title & $\begin{array}{l}\text { A New Model-Free Feature Screening Procedure for } \\
\text { Ultrahigh-Dimensional Interval-Censored Failure Time } \\
\text { Data }\end{array}$ \\
\hline Manuscript ID & SS-2020-0174 \\
\hline URL & http://www.stat.sinica.edu.tw/statistica/ \\
\hline DOI & $10.5705 /$ ss.202020.0174 \\
\hline Complete List of Authors & $\begin{array}{l}\text { Jing Zhang, } \\
\text { Mingyue Du, } \\
\end{array}$ \\
& $\begin{array}{l}\text { Yanyan Liu and } \\
\text { Jianguo Sun }\end{array}$ \\
\hline Corresponding Author & Yanyan Liu \\
\hline E-mail & liuyy@whu.edu.cn \\
\hline Notice: Accepted version subject to English editing. \\
\hline
\end{tabular}


Statistica Sinica

\title{
A NEW MODEL-FREE FEATURE SCREENING PROCEDURE FOR ULTRAHIGH-DIMENSIONAL INTERVAL-CENSORED FAILURE TIME DATA
}

\author{
Jing Zhang, Mingyue Du, Yanyan Liu* and Jianguo Sun
}

Zhongnan University of Economics and Law

The Hong Kong Polytechnic University

Wuhan University and University of Missouri

\begin{abstract}
Screening important features based on ultrahigh-dimensional data has become one of the important tasks in statistical analysis, and correspondingly, various screening procedures have been proposed for various types of studies or data including complete data and right-censored failure time data. In this paper, we consider ultrahigh-dimensional interval-censored failure time data, which frequently occur in medical follow-up studies among others and include rightcensored data as a special case but for which only limited work exists. For the problem, a distance correlation-based sure independent screening procedure is proposed, and the new approach is model-free and does not require the estimation of survival functions unlike most of the existing nonparametric screening procedures for failure time data. We establish the sure screening property and the ranking consistency of the proposed method and conduct an extensive simu-
\end{abstract}


lation study, which suggests that the proposed procedure works well for practical situations. Also we apply it to a set of real data on Alzheimer's Disease that motivated this study.

Key words and phrases: Distance correlation, interval-censored data, model-free screening, sure screening property, ultrahigh-dimensional data.

\section{Introduction}

Screening important features based on ultrahigh-dimensional data has become one of the important tasks in statistical analysis, and correspondingly, various screening procedures have been proposed. For example, one of the early work was given by Fan and Lv (2008), who proposed a sure independence screening (SIS) procedure under the framework of linear regression model. Following them, many authors extended the SIS procedure to different models, including the generalized linear model (Fan and Song, 2010), the additive model (Fan, Feng and Song, 2011), the multi-index model (Zhu et al., 2011), and Li, Zhong and Zhu (2012) gave a distance correlationbased SIS procedure. In this paper, we will discuss the same problem but unlike the work mentioned above, we will consider a type of incomplete data, interval-censored (IC) failure time data (Sun, 2006). By IC data, we mean that the failure time of interest is known or observed to belong to an interval instead of being observed exactly, and it is easy to see that 
such data can commonly occur in many fields with periodic-ups, especially medical studies such as clinical studies. Also it is not hard to see that IC data include right-censored data as a special case and their analysis is much difficult than the analysis of the latter due to their much more complicated structures.

An example of IC data that motivated this study is that arising from the Alzheimer's Disease Neuroimaging Initiative, a longitudinal follow-up study designed to develop clinical, imaging, genetic, and biochemical biomarkers for the early detection and tracking of the Alzheimer's disease (AD). In the study, among others, one variable of interest is the AD conversion time, defined as the time from the baseline visit date to the AD conversion. Since the participants were examined intermittently or periodically, the exact time of the $\mathrm{AD}$ conversion is not available and instead only IC data are available on the variable. In other words, if happening, the occurrence of the $\mathrm{AD}$ conversion is only known to be within an interval. On the covariates or factors that are of interest and for which information was collected, the study consists of the information on a large number of single nucleotide polymorphisms (SNPs) for each participant, with the aim being to detect the SNPs that have significant effects on the risk of developing the AD. For example, one question of interest can be which of the SNPs are individually 
most associated with the AD conversion and another is how one can predict the AD conversion by using the SNPs and other information.

Many authors have considered the generalizations of the SIS procedure to the screening of important features based on right-censored failure time data. In general, the developed procedures can be classified into two types, model-based ones (Tibshirani, 2009; Fan, Feng and Wu, 2010; Zhao and Li, 2012; Gorst-Rasmussen and Scheike, 2013) and modelfree methods (Song et al., 2014; Wu and Yin, 2015; Zhang, Liu and Wu, 2017; Zhou and Zhu, 2017; Liu, Zhang and Zhao, 2018; Zhang et al., 2018; Lin, Liu and Hao, 2018; Zhang, Liu and Cui, 2020). However, limited SIS methods have been studied for IC failure time data. Note that one simple generalization is to consider the procedures developed for right-censored data that involve the nonparametric estimation of survival functions and to simply replace the estimators by the Turnbull's estimator for IC data (Turnbull, 1976). One such method, the only existing screening method for IC failure time data, is that given in $\mathrm{Hu}$ et al. (2020), who generalized the screening method for right-censored data proposed by Zhang et al. (2018) to case II IC data. In addition to others, as discussed in more details below, one drawback of such approach is the huge computational burden since there is no closed form for the Turnbull's estimator. 
Several regularized methods have been proposed in the literature for the variable or covariate selection based on high dimensional IC data. For example, Wu and Cook (2015) proposed such a procedure for the proportional hazards model with the baseline hazard function being a piecewise constant function and gave an EM algorithm for maximizing the penalized log-likelihood function. Also Scolas et al. (2016) extended the adaptive Lasso procedure under a flexible parametric mixture cure model structure, and Zhao et al. (2020) proposed a broken adaptive ridge regression procedure that combines the strengths of the quadratic regularization and the adaptive weighted bridge shrinkage. However, these methods cannot apply or would face the simultaneous challenges of computational expediency, statistical accuracy and algorithmic stability if the dimension or the number of covariates $p$ is ultrahigh in the sense that $p=\exp \left(n^{\alpha}\right)$ with $n$ denoting the sample size and $\alpha>0$ (Fan, Samworth and Wu, 2009).

In the following, we will propose a SIS procedure for ultrahigh-dimensional IC failure time data by employing the distance correlation (DC) between a redefined response and each predictor as the dependence measure. As pointed out by Székely, Rizzo and Bakirov (2007), the DC of two random vectors equals to zero if and only if they are independent and therefore could be used as a sensitive dependence measure. One major advantage of the 
proposed approach is that it does not involve the nonparametric estimation of a survival function or any complicated numerical optimization and thus it is easy to implement and converges fast. Also it is model-free and thus robust to model misspecification. In addition, it applies to general and complex IC data and thus also the mixture of left-, interval-, and right-censored data, and the large sample properties of the proposed method, including the sure screening property and the rank consistency, will be established.

The remainder of the article is organized as follows. First we will introduce some notations and assumptions that will be used throughout the paper in Section 2 and also review some background about the DC. The proposed model-free screening procedure will be presented in Section 3, and in Section 4 we will establish the theoretical properties of the proposed approach. Section 5 will present some results obtained from simulation studies conducted to evaluate the finite sample performance of the method and they indicate that it works well for practical situations. To determine the selection threshold value for the proposed screening procedure, we propose and investigate the performance of a data-driven log-ratio criterion in Section 6, and Section 7 applies the method to the AD example discussed above. Some discussion and concluding remarks are provided in Section 8. 


\section{Notations, Assumptions and Distance Correlation}

Consider a failure time study that consists of $n$ independent subjects and let $T$ denote the failure time of interest. For each $T$ or subject, suppose that there exist two monitoring or observation times denoted by $U$ and $V$ with $U<V$ and $T$ is observed only to be in one of three situations. They are that $T$ is between $U$ and $V$ or interval-censored, $T$ is larger than $V$ or right-censored, or $T$ is less than $U$ or left-censored. That is, only IC data are available on $T$ or more specifically, they are often referred to as case II IC data (Sun, 2006). Define the indicator variables $\Delta_{1}=I(T<U)$, $\Delta_{2}=I(U \leq T<V)$ and $\Delta_{3}=1-\Delta_{1}-\Delta_{2}$, and let $T_{i}, U_{i}, V_{i}, \Delta_{1 i}$, $\Delta_{2 i}$ and $\Delta_{3 i}$ denote the $T, U, V, \Delta_{1}, \Delta_{2}$ and $\Delta_{3}$ defined above associated with subject $i(i=1, \ldots, n)$. Then the observed data on the $T_{i}$ 's can be summarized as $\left\{U_{i}, V_{i}, \Delta_{1 i}, \Delta_{2 i}, \Delta_{3 i}: i=1,2, \ldots, n\right\}$.

For each study subject, suppose that there exists a $p$-dimensional vector of covariates denoted by $\boldsymbol{Z}=\left(Z_{1}, \ldots, Z_{p}\right)^{\mathrm{T}}$ and $p$ can be ultrahigh. Let $S(t \mid \boldsymbol{Z})=P(T>t \mid \boldsymbol{Z})$ denote the survival function for a subject with the covariate $\boldsymbol{Z}$ and by following Song et al. (2014) and others, define

$$
\mathcal{A}=\left\{k: S(t \mid \boldsymbol{Z}) \text { functionally depends on } Z_{k} \text { for } t \geq 0, k=1, \ldots, p\right\}
$$

the index set of the active covariates or the covariates that have some effects on $T$. To understand the definition above, let $\boldsymbol{Z}_{\mathcal{A}}$ denote the sub- 
vector of $\boldsymbol{Z}$ containing all of the active covariates or the components in $\mathcal{A}$. Then $\mathcal{A}$ means that $S(t \mid \boldsymbol{Z})$ depends on $\boldsymbol{Z}$ only through $\boldsymbol{Z}_{\mathcal{A}}$ or $S(t \mid \boldsymbol{Z})=$ $S\left(t \mid \boldsymbol{Z}_{\mathcal{A}}\right)$ for any $t$. Suppose that the goal is to determine or estimate $\mathcal{A}$ through a screening procedure. Since our proposed screening utility is based on distance correlation (DC), we first introduce the concept of DC briefly. Let $\boldsymbol{u}$ and $\boldsymbol{v}$ denote two random vectors, and $\phi_{\boldsymbol{u}, \boldsymbol{v}}(t, s), \phi_{\boldsymbol{u}}(t)$ and $\phi_{\boldsymbol{v}}(s)$ be the characteristic functions of $(\boldsymbol{u}, \boldsymbol{v}), \boldsymbol{u}$ and $\boldsymbol{v}$, respectively. The DC between $\boldsymbol{u}$ and $\boldsymbol{v}$ is defined as

$$
\operatorname{dcorr}^{2}(\boldsymbol{u}, \boldsymbol{v})=\frac{\operatorname{dcov}^{2}(\boldsymbol{u}, \boldsymbol{v})}{\sqrt{\operatorname{dcov}^{2}(\boldsymbol{u}, \boldsymbol{u}) \operatorname{dcov}^{2}(\boldsymbol{v}, \boldsymbol{v})}}
$$

where $\operatorname{dcov}^{2}(\boldsymbol{u}, \boldsymbol{v})$ denotes the distance covariance between $\boldsymbol{u}$ and $\boldsymbol{v}$ and is given by

$$
\mathrm{d}_{\operatorname{cov}^{2}}(\boldsymbol{u}, \boldsymbol{v})=\frac{1}{c_{d_{u}} c_{d_{v}}} \int_{R^{d_{u}+d_{v}}} \frac{\left\|\phi_{\boldsymbol{u}, \boldsymbol{v}}(t, s)-\phi_{\boldsymbol{u}}(t) \phi_{\boldsymbol{v}}(s)\right\|^{2}}{\|t\|_{d_{u}}^{1+d_{u}}\|s\|_{d_{v}}^{1+d_{v}}} \mathrm{~d} t \mathrm{~d} s
$$

In the above,

$$
c_{d_{u}}=\pi^{\left(1+d_{u}\right) / 2} / \Gamma\left(\frac{1+d_{u}}{2}\right), c_{d_{v}}=\pi^{\left(1+d_{v}\right) / 2} / \Gamma\left(\frac{1+d_{v}}{2}\right),
$$

where $\Gamma(\cdot)$ denotes the Gamma function and $d_{u}$ and $d_{v}$ denote the dimensions of $\boldsymbol{u}$ and $\boldsymbol{v}$, respectively. Székely, Rizzo and Bakirov (2007) proved that $\operatorname{dcorr}^{2}(\boldsymbol{u}, \boldsymbol{v})=0$ if and only if $\boldsymbol{u}$ and $\boldsymbol{v}$ are independent. This suggests that the DC could be used as a sensitive measure of dependence, and 
Li, Zhong and Zhu (2012) did develop a DC-based screening procedure for the complete data situation. In the following, we focus on case II intervalcensored data.

\section{Distance Correlation Screening Procedure}

As discussed above, a natural screening utility based on DC is $\operatorname{dcorr}^{2}\left(Z_{k}, T\right)$, the DC between $Z_{k}$ and $T$. However, $\operatorname{dcorr}^{2}\left(Z_{k}, T\right)$ cannot be directly estimated with the incomplete IC data. Consider a more general data including interval-censored, right-censored and left-censored observations. Note that both right-censored and left-censored observations could be viewed as special cases of interval-censored observations. Define two new variables $L$ and $H$, representing the length and endpoint of the time interval within which the event time lies, respectively, as

$$
\begin{aligned}
L & =\Delta_{1} U+\Delta_{2}(V-U)+\Delta_{3}(\eta-V), \\
H & =\Delta_{1} \cdot U+\Delta_{2} \cdot V+\Delta_{3} \cdot V
\end{aligned}
$$

where $\eta$ is a large constant. In practice, any large number can be used for $\eta$ such as $\eta=10^{6}$, which is used in the numerical studies below. Note that in the above, $H=V$ if $T$ is either between $U$ and $V$ (interval-censored) or larger than $V$ (right-censored), and $H=U$ if $T$ is less than $U$ (leftcensored). In other words, $H$ represents either the left or right endpoint of 
the observed interval. According to Székely, Rizzo and Bakirov (2007), the following four conditions are equivalent:

(1) $\operatorname{dcorr}^{2}\left(Z_{k},(L, H)\right)=0$;

(2) $(L, H)$ and $Z_{k}$ are independent;

(3) $(a \cdot L+b, c \cdot H+d)$ and $Z_{k}$ are independent for any constants $a, c \neq 0$ and constants $b, d$;

(4) $\operatorname{dcorr}^{2}\left(Z_{k},(a \cdot L+b, c \cdot H+d)\right)=0$ for any constants $a, c \neq 0$ and constants $b, d$.

Note that if $\operatorname{dcorr}^{2}\left(Z_{k},(L, H)\right)>0$, the value of $\operatorname{dcorr}^{2}\left(Z_{k},(a \cdot L+b, c\right.$. $H+d)$ ) depends on $a$ and $c$ when $a \neq c$. To eliminate the influence of $a$ and $c$, we first standardize $(L, H)$ marginally as follows.

$$
L^{*}=\frac{L-\mu_{1}}{\sigma_{1}}, H^{*}=\frac{H-\mu_{2}}{\sigma_{2}},
$$

where $\mu_{1}=\mathrm{E}(L), \mu_{2}=\mathrm{E}(H), \sigma_{1}=\operatorname{sd}(L), \sigma_{2}=\operatorname{sd}(H)$, and $\mathrm{E}(\cdot)$ and $\operatorname{sd}(\cdot)$ represent the expectation and standard deviation of the corresponding variable, respectively. Define $\boldsymbol{Y}=\left(L^{*}, H^{*}\right)$ and

$$
\omega_{k}=\operatorname{dcorr}^{2}\left(Z_{k}, \boldsymbol{Y}\right)=\frac{\operatorname{dcov}^{2}\left(Z_{k}, \boldsymbol{Y}\right)}{\sqrt{\operatorname{dcov}^{2}\left(Z_{k}, Z_{k}\right) \operatorname{dcov}^{2}(\boldsymbol{Y}, \boldsymbol{Y})}}
$$


which will serve as the population quantity of the proposed marginal utility measure for ranking the dependence between the covariate $Z_{k}$ and the failure time $T$.

A key feature of $\omega_{k}=\operatorname{dcorr}^{2}\left(Z_{k}, \boldsymbol{Y}\right)$ defined above is that unlike dcorr ${ }^{2}\left(Z_{k},(a\right.$. $L+b, c \cdot H+d)), \operatorname{dcorr}^{2}\left(Z_{k},\left(a \cdot L^{*}+b, c \cdot H^{*}+d\right)\right)$ does not depend on $a$ and $c$. More discussions on their comparison will be given below. Based on remark 3 in Székely, Rizzo and Bakirov $(2007), \operatorname{dcov}^{2}\left(Z_{k}, \boldsymbol{Y}\right)$ can be partitioned as

$$
\operatorname{dcov}^{2}\left(Z_{k}, \boldsymbol{Y}\right)=S_{k 1}+S_{k 2}-2 S_{k 3}
$$

In the above, $S_{k 1}=\mathrm{E}\left(\left\|Z_{k}-\widetilde{Z}_{k}\right\|_{1}\|\boldsymbol{Y}-\widetilde{\boldsymbol{Y}}\|_{2}\right), S_{k 2}=\mathrm{E}\left(\left\|Z_{k}-\widetilde{Z}_{k}\right\|_{1}\right) \mathrm{E}(\| \boldsymbol{Y}-$ $\left.\tilde{\boldsymbol{Y}} \|_{2}\right)$, and $S_{k 3}=\mathrm{E}\left\{\mathrm{E}\left(\left\|Z_{k}-\widetilde{Z}_{k}\right\|_{1} \mid Z_{k}\right) \mathrm{E}\left(\|\boldsymbol{Y}-\tilde{\boldsymbol{Y}}\|_{2} \mid \boldsymbol{Y}\right)\right\}$ with $\left(\widetilde{Z}_{k}, \widetilde{\boldsymbol{Y}}\right)$ denoting an independent copy of $\left(Z_{k}, \boldsymbol{Y}\right)$ and $\|\cdot\|_{1}$ and $\|\cdot\|_{2}$ the Euclidean norm.

Let $\left(L_{i}, H_{i}\right), i=1, \cdots, n$ be a random sample from $(L, H)$. Let $\left(\widehat{\mu}_{1}, \widehat{\sigma}_{1}\right)$ and $\left(\widehat{\mu}_{2}, \widehat{\sigma}_{2}\right)$ denote the (sample mean, sample standard error) of the $L_{i}$ 's and $H_{i}$ 's, respectively. This yields a standardized data $\left\{\widehat{\boldsymbol{Y}}_{i}=\left(\widehat{L}_{i}^{*}, \widehat{H}_{i}^{*}\right)\right.$ : $i=1, \ldots, n\}$, where

$$
\widehat{L}_{i}^{*}=\frac{L_{i}-\widehat{\mu}_{1}}{\widehat{\sigma}_{1}}, \widehat{H}_{i}^{*}=\frac{H_{i}-\widehat{\mu}_{2}}{\widehat{\sigma}_{2}} .
$$

Then the empirical estimator of $\operatorname{dcov}^{2}\left(Z_{k}, \boldsymbol{Y}\right)$ is given by

$$
\widehat{\operatorname{dcov}}^{2}(Z k, \boldsymbol{Y})=\widehat{S}_{k 1}+\widehat{S}_{k 2}-2 \widehat{S}_{k 3},
$$


where

$$
\begin{aligned}
& \widehat{S}_{k 1}=1 / n^{2} \sum_{i=1}^{n} \sum_{j=1}^{n}\left|Z_{k i}-Z_{k j}\right|\left\|\widehat{\boldsymbol{Y}}_{i}-\widehat{\boldsymbol{Y}}_{j}\right\|_{2}, \\
& \widehat{S}_{k 2}=1 / n^{2} \sum_{i=1}^{n} \sum_{j=1}^{n}\left|Z_{k i}-Z_{k j}\right| \cdot 1 / n^{2} \sum_{i=1}^{n} \sum_{j=1}^{n}\left\|\widehat{\boldsymbol{Y}}_{i}-\widehat{\boldsymbol{Y}}_{j}\right\|_{2}, \\
& \widehat{S}_{k 3}=1 / n^{3} \sum_{i=1}^{n} \sum_{j=1}^{n} \sum_{l=1}^{n}\left|Z_{k i}-Z_{k l}\right| \cdot\left\|\widehat{\boldsymbol{Y}}_{j}-\widehat{\boldsymbol{Y}}_{l}\right\|_{2} .
\end{aligned}
$$

Similarly, we can obtain the empirical estimators of $\widehat{\operatorname{dcov}}^{2}\left(Z_{k}, Z_{k}\right)$ and $\widehat{\operatorname{dcov}}^{2}(\boldsymbol{Y}, \boldsymbol{Y})$ and accordingly an empirical estimator of $\omega_{k}$

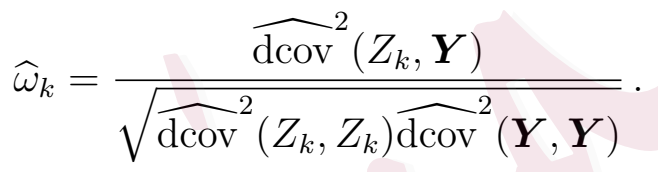

Based on the discussion above and the property of distance correlation (Székely, Rizzo and Bakirov, 2007), the estimator $\widehat{\omega}_{k}$ is expected to fluctuate around zero if $Z_{k}$ is an inactive covariate and be away from zero otherwise. In other words, we can select those candidate covariates with top values of $\widehat{\omega}_{k}$ as active covariates. This motivates the estimate of $\mathcal{A}$ or the screening procedure given by

$$
\widehat{\mathcal{A}}=\left\{k: \widehat{\omega}_{k} \geq c n^{-\kappa}, k=1, \ldots, p\right\}
$$

for some pre-specified threshold constants $c$ and $\kappa$ to be discussed below.

In practice, it is difficult to obtain the constants $c$ and $\kappa$ or the cutoff threshold $c n^{-\kappa}$ which is used to separate the active and inactive set. 
Following the thresholding rule by Fan and Lv (2008), we instead propose to rank $\left\{\widehat{\omega}_{k}, k=1, \ldots, p\right\}$ from the largest to smallest and to select the covariates based on the top ones or estimate $\mathcal{A}$ by

$$
\widetilde{\mathcal{A}}=\left\{j: \widehat{\omega}_{j} \text { is amongst the first } d_{0} \text { largest of all } \widehat{\omega}_{k}, k=1, \ldots, p\right\} .
$$

In the above, $d_{0}$ is a pre-determined positive integer and suggested to be $d_{0}=\lceil n / \log n\rceil$ by following Fan and Lv (2008). This choice of $d_{0}$ has been widely adopted in the existing literature of screening procedure (e.g., Zhu et al., 2011; Li, Zhong and Zhu, 2012; Song et al., 2014). More discussion on this will be given below along with a new maximum log-ratio criterion.

\section{Asymptotic Properties}

In this section, we establish the sure screening and ranking consistency properties of the model-free screening procedure proposed in the previous sections. For this, we need the following conditions.

C1. There exists a positive constant $s_{0}$ such that for all $0<s \leq 2 s_{0}$, we have that

$$
\sup _{p} \max _{1 \leq k \leq p} E\left\{\exp \left(s\|G\|_{1}^{2}\right)\right\}<\infty
$$

for $G=Z_{k}$ and $\tilde{V}$ with $\tilde{V}=\max \{U, V\}, V$ or $U$ for interval-censored, right-censored or left-censored observations, respectively. 
C2. The minimum distance correlation of active predictors satisfies $\min _{k \in \mathcal{A}} \omega_{k} \geq$ $2 c n^{-\kappa}$ for some constants $c>0$ and $\kappa \in[0,1 / 2)$.

Condition $\mathrm{C} 1$ is a common assumption required by most of the existing screening procedures. Condition $\mathrm{C} 2$ requires that the values of marginal utilities between each active variable and response are not too small. It is a typical assumption in the literature of feature screening and similar to condition 3 of Fan and Lv (2008), condition 2 of Li, Zhong and Zhu (2012), and conditions 2 and 5 of $\mathrm{Wu}$ and Cook (2015) among others. We will first present the sure screening property of the proposed screening method and then the ranking consistency property.

Theorem 1. Under condition C1, for any $0<\gamma<1 / 2-\kappa$, there exist positive constants $c_{1}>0$ and $c_{2}>0$ such that

$P\left(\max _{1 \leq k \leq p}\left|\widehat{\omega}_{k}-\omega_{k}\right| \geq c n^{-\kappa}\right) \leq O\left(p\left[\exp \left\{-c_{1} n^{1-2(\kappa+\gamma)}\right\}+n \exp \left(-c_{2} n^{\gamma}\right)\right]\right)$. Also under conditions $C 1$ and C2, we have that

$$
P(\mathcal{A} \subseteq \widehat{\mathcal{A}}) \geq 1-O\left(a\left[\exp \left\{-c_{1} n^{1-2(\kappa+\gamma)}\right\}+n \exp \left(-c_{2} n^{\gamma}\right)\right]\right)
$$

where $a=|\mathcal{A}|$ is the cardinality of $\mathcal{A}$.

Theorem 2. Assume $\omega_{k}=0$ holds for $k \notin \mathcal{A}$. Then under conditions $C 1$ 
and C2, there exist positive constants $c_{1}>0$ and $c_{2}>0$ such that $P\left(\max _{k \notin \mathcal{A}} \widehat{\omega}_{k}<\min _{k \in \mathcal{A}} \widehat{\omega}_{k}\right) \geq 1-O\left(p\left[\exp \left\{-c_{1} n^{1-2(\kappa+\gamma)}\right\}+n \exp \left(-c_{2} n^{\gamma}\right)\right]\right)$

The proof of the results given above is sketched in the supplementary material. It is worth noting that although the idea used here is similar to that discussed in $\mathrm{Li}$, Zhong and Zhu (2012), the proof here is much more complicated. One main reason is that unlike Li, Zhong and Zhu (2012), we have to estimate the redefined response $\boldsymbol{Y}=\left(L^{*}, H^{*}\right)$ in addition to estimate the distance correlation $\operatorname{dcorr}^{2}\left(Z_{k}, \boldsymbol{Y}\right)$. More specifically, among others, we have to obtain the exponential tail probability bound for $P\left(\left|\operatorname{dcov}^{2}\left(Z_{k}, \boldsymbol{Y}\right)-\widehat{\operatorname{dcov}}^{2}\left(Z_{k}, \boldsymbol{Y}\right)\right| \geq \epsilon\right)$ in order to prove Theorem 1. For this, one needs to compute $P\left(\left|\operatorname{dcov}^{2}\left(Z_{k}, \boldsymbol{Y}\right)-\widetilde{\operatorname{dcov}}^{2}\left(Z_{k}, \boldsymbol{Y}\right)\right| \geq \epsilon\right)$ and $P\left(\left|\widehat{\operatorname{dcov}}^{2}\left(Z_{k}, \boldsymbol{Y}\right)-\widetilde{\operatorname{dcov}}^{2}\left(Z_{k}, \boldsymbol{Y}\right)\right| \geq \epsilon\right)$. The calculation of the former can be obtained in the same way as Li, Zhong and Zhu (2012) but the determination of the latter is completely new. More details can be found in the Supplementary Material.

Theorem 1 guarantees that the proposed DC-based SIS will retain the active set $\mathcal{A}$, and Theorem 2 shows the reasonability or validity of the proposed dependence measure. Together the results above state that if 
$p=\exp \left\{n^{\alpha}\right\}$ with $0 \leq \alpha<(1-2 \kappa) / 3$, we have that

$$
\lim _{n \rightarrow \infty} P(\mathcal{A} \subseteq \widehat{\mathcal{A}})=1, \quad \lim _{n \rightarrow \infty} P\left(\max _{k \notin \mathcal{A}} \widehat{\omega}_{k}<\min _{k \in \mathcal{A}} \widehat{\omega}_{k}\right)=1
$$

This proves that the DC values of all active predictors can be expected to be larger than those of all inactive variables asymptotically and all active covariates can be selected with probability approaching to one as $n \rightarrow \infty$. In other words, it is reasonable to choose the covariates or predictors for which the $\omega_{k}$ 's are among the $d_{0}$ largest ones.

\section{Simulation Studies}

To assess the finite sample performance of the DC-based SIS procedure proposed in the previous sections, a sequence of simulation studies were conducted under different model settings and distributional assumptions of the predictor variables. Note that instead of the proposed approach, a naive alternative method is to directly set $Y=\left(U, V, \Delta_{1}, \Delta_{2}, \Delta_{3}\right)$ and then build the marginal utility $\omega_{k}$ as did above. For comparison, we also considered this alternative procedure, which is referred to as DC-SIS1, while the proposed method is referred to as DC-SIS. For measuring the performance, following Li, Zhong and Zhu (2012), we considered the following quantities.

(i) $\mathcal{S}$ : the minimum model size required to include all active variables. In the tables below, the average value of $\mathcal{S}$ and a robust estimate of 
its standard deviation, denoted as MMS and RSD, will be reported, where RSD is defined as IQR/1.34 with IQR denoting the interquartile range of $\mathcal{S}$ over all replications (Huang and Zhu, 2016).

(ii) $\mathcal{P}_{e}$ : the selection proportion that each active variable is selected into the model with the model size $d_{0}=\lceil n / \log n\rceil$, where $\lceil x\rceil$ denotes the integer part of $x$.

(iii) $\mathcal{P}_{a}$ : the selection proportion that all active variables are selected into the model with the model size $d_{0}$ as above.

It is apparent that an effective screening procedure is expected to yield $\mathcal{S}$ close to the true minimum model size and both $\mathcal{P}_{e}$ and $\mathcal{P}_{a}$ close to one.

For the generation of observed data, for subject $i$, we first randomly generated a sequence of observation times from the uniform distribution $\mathrm{U}(0, \tau)$, denoted by $t_{1 i}<t_{2 i}<\ldots<t_{m i}$. If $T_{i}<t_{1 i}$, we set the censoring type for subject $i$ as left-censored, and if $T_{i}>t_{m i}$, we set the censoring type for subject $i$ as right-censored. Otherwise, we set the censoring type as interval-censored and $U_{i}$ and $V_{i}$ to be the largest observation time that is smaller than $T_{i}$ and the smallest observation time that is greater than $T_{i}$, respectively. The constant $\tau$, which controls the percentages of leftcensored, interval-censored and right-censored observations, was chosen to 
yield $20 \%, 60 \%$ and $20 \%$. The failure time of interest was assumed to follow the Cox proportional hazards model, the nonlinear model or the general transformation model as described below. The results given below are based on $p=2000$ or $4000, n=100$ or 200 , and $\eta=10^{6}$ with 500 replications.

Set-up 1. In this case, the failure time of interest $T_{i}$ was generated from the Cox proportional hazards model given by

$$
\lambda\left(t \mid \boldsymbol{Z}_{i}\right)=\lambda_{0}(t) \exp \left(\boldsymbol{Z}_{i}^{\mathrm{T}} \boldsymbol{\beta}_{0}\right)
$$

where $\lambda_{0}(t)=(t-0.5)^{2}$ and $\boldsymbol{Z} \sim N_{p}(\mathbf{0}, \boldsymbol{\Sigma})$ with $\boldsymbol{\Sigma}=\left(0.8^{|i-j|}\right)$ for $i, j=$ $1, \ldots, p$. Here we set $\boldsymbol{\beta}_{0}=(1,0.8,0.6,0.4,0.2,0, \ldots, 0)^{\mathrm{T}}$, i.e., there are five active covariates, and the index set of active covariates is $\mathcal{A}=\{1,2,3,4,5\}$. Table 1 presents the simulation results of MMS, RSD, $\mathcal{P}_{e}$ and $\mathcal{P}_{a}$ for these two procedures, from which we can see the proposed procedure DC-SIS performs well for all cases and is clearly better than DC-SIS1, especially for small sample size situations.

Set-up 2. For the situation, we generated the failure time of interest from the nonlinear survival model with interactions given by

$$
T=\left(2+\sin Z_{1}\right)^{2}+0.5\left(1+Z_{5}\right)^{-3}+3\left(Z_{10}^{2}+Z_{10}\right)+0.5 Z_{1} Z_{10}+\epsilon
$$

where $\epsilon \sim N(0,1)$. Note that here we have three active covariates in $Z_{1}$, $Z_{5}$ and $Z_{10}$, giving the index set of the active covariates $\mathcal{A}=\{1,5,10\}$. For 
covariates, we considered three situations.

a) $\boldsymbol{Z} \sim N_{p}(\mathbf{0}, \boldsymbol{\Sigma})$ with $\boldsymbol{\Sigma}=\left(0.8^{|i-j|}\right)$ for $i, j=1, \ldots, p$;

b) The first component $Z_{1}$ was generated from the Bernoulli distribution with success probability 0.5 and the remaining $(p-1)$ components $\left(Z_{2}, \ldots, Z_{p}\right)$ were assumed to follow the multivariate normal distribution $N_{p}(\mathbf{0}, \mathbf{\Sigma})$ with $\boldsymbol{\Sigma}=\left(0.8^{|i-j|}\right)$ for $i, j=1, \ldots,(p-1)$.

c) All covariates $Z_{k}(k=1, \ldots, p)$ were generated from the Bernoulli distribution with the success probability 0.5 , independently.

Table 2 gives the simulation results, containing the same quantities as in Table 1 . The results again indicate that the proposed screening procedure seems to give reasonable performance for the complicated nonlinear model with interactions. In particular, the procedure appears to perform better for the continuous covariate than for the categorical covariate, but the difference between the types of covariates decreased when the sample size increased. In other words, the proposed method seems to give good performance for both continuous and categorical covariates when the sample size is large. Furthermore, as seen in Table 2, the proposed procedure DCSIS performs overwhelmingly superior to the naive DC-based SIS procedure DC-SIS1 for the complicated nonlinear model, especially for the cases with 
categorical covariates.

To evaluate the performance of the proposed procedure under different observation schedules, we considered another set-up. More specially, we first randomly generated a sequence of observation times from the uniform distribution $\mathrm{U}(0, \tau)$ denoted by $t_{1}<t_{2}<\ldots<t_{m}$. For each subject $i$ and at each observation time $t_{j}$, a random variable was then independently generated from the Bernoulli distribution with the success probability 0.6. If the value is 1 , it was assumed that subject $i$ is observed at $t_{j}$ and otherwise, not observed. In addition to the percentages of left-, right-, interval-censored observations being 20\%,20\%,60\%, we also considered the cases with the percentages being $20 \%, 60 \%, 20 \%$ or $60 \%, 20 \%, 20 \%$, and denote them as cases I, II and III, respectively. Table 3 gives the results obtained with $m=20$ and under case a), and they suggest that the proposed method again performs well for the situation. Also as expected, the results indicate that the proposed method seems to be robust with respect to the percentages of left-, right-, and interval-censored observations.

Set-up 3. In this set-up, we generated the failure time of interest $T_{i}$ from the transformation model

$$
H(T)=-\boldsymbol{\beta}_{0}^{\mathrm{T}} \boldsymbol{Z}+\epsilon,
$$

where we took $H(t)=\log \left\{0.5\left(e^{2 t}-1\right)\right\}, \boldsymbol{\beta}_{0}=\left(1,0.7, \mathbf{0}_{6}, 0.8,1.0, \mathbf{0}_{p-10}\right)^{\mathrm{T}}$, 
and $\boldsymbol{Z} \sim N_{p}(\mathbf{0}, \boldsymbol{\Sigma})$ with $\boldsymbol{\Sigma}=\left(0.5^{|i-j|}\right)$ for $i, j=1, \ldots, p$. Here we have four active covariates in $Z_{1}, Z_{2}, Z_{9}$ and $Z_{10}$, giving the index set of the active covariates $\mathcal{A}=\{1,2,9,10\}$. For the distribution of $\epsilon$, we considered three choices and they are the standard normal distribution, the standard logistic distribution and the type-I extreme value distribution. The obtained simulation results on the same quantities as above are presented in Table 4, and they again suggest that the proposed procedure DC-SIS gave satisfactory results and seems to be robust with respect to the error distribution. Also again the proposed procedure DC-SIS gave much better performances than the DC-SIS1 procedure.

To further assess the performance of the proposed screening procedure in terms of $\mathcal{S}$, Figure 1 gives the box plots of $\mathcal{S}$ obtained based on 500 replications under the three set-ups discussed above for the left-censored, right-censored, interval-censored rates being $(20 \%, 20 \%, 60 \%)$. Again they indicate that the proposed procedure gives excellent performance and can screen out the inactive covariates as expected.

\section{A Data-driven Log-ratio Criterion}

As discussed above, to apply the proposed screening procedure as well as other existing screening procedures, one needs to choose a selection thresh- 
old value and for this, a common choice was given by Fan and Lv (2008), who suggested a hard cutoff value $d_{0}=\lceil n / \log n\rceil$. In general, the use of this choice relies on the sparsity assumption, meaning that only a small number of variables or covariates are truly associated with the response variable of interest, which is often true in many situations such as gene selection and biomedical imaging data analysis. On the other hand, it is apparent that sometimes the sparsity assumption may not hold and also it will be useful to develop a data-driven criterion for the choice. Based on Theorem 2, we propose the following log-ratio criterion.

Let $\widehat{\omega}_{k}$ 's be defined as above and $\widehat{\omega}_{(k)}$ denote the $k$ th largest of all $\left\{\widehat{\omega}_{k}\right.$ : $k=1, \ldots, p\}$. Consider the sequence $\left\{\log \left(\widehat{\omega}_{(k)} / \widehat{\omega}_{(k+1)}\right)\right\}$. In general, one can expect that sometimes the sequence will increase and sometimes may decrease when $k$ corresponds to active covariates. Also it tends to give the largest value when $k$ approaches the boundary between active and inactive covariates and then decreases among inactive covariates. This motivates the following log-ratio criterion for choosing the selection threshold value

$$
d_{0}^{*}=\operatorname{argmax}_{1 \leq j \leq(p-1)} \log \frac{\widehat{\omega}_{(k)}}{\widehat{\omega}_{(k+1)}} .
$$

To investigate the behave of the sequence defined above and assess the performance of the log-ratio criterion, we repeated the simulation study discussed above under the set-up 1 with $n=100, p=2000$, and the true 
model size being $p_{0}=5,10,20,50$ or 100 . Figure 2 plots the first 200 $\widehat{\omega}_{(k)}$ 's obtained from $\left\{\widehat{\omega}_{k}: k=1, \ldots, p\right\}$ with each $\widehat{\omega}_{k}$ being the average value over 500 replications, and Figure 3 gives the plot of the corresponding $\log \left(\widehat{\omega}_{(k)} / \widehat{\omega}_{(k+1)}\right)$. Note that in both figures, the solid points denote the active covariates. One can see from Figure 2 that all of the active covariates are located on the left side of the inactive covariates and there seems to exist a change point from which the value of $\omega$ goes smoothly. Figure 3 indicates that the ratio $\log \left(\widehat{\omega}_{(k)} / \widehat{\omega}_{(k+1)}\right)$ indeed behaves as described above and confirms the effectiveness of the proposed log-ratio criterion.

\section{An Application}

In this section, we apply the model-free DC-based screening procedure proposed in the previous sections to the data arising from the Alzheimer's Disease Neuroimaging Initiative (ADNI) discussed before. As said above, the participants in the study were examined intermittently for various factors including demographic and clinical factors and SNPs and are classified into three groups based on their cognitive conditions, cognitively normal, mild cognitive impairment and Alzheimer's disease (AD). Among others, one variable of interest is the time (in years) from the baseline visit date to the $\mathrm{AD}$ conversion, and due to the design of the study, only interval- 
censored data are available on the AD conversion time. In the following, we are interested in identifying the SNPs that have significant effects on the $\mathrm{AD}$ conversion time.

In the analysis below, by following others (Li et al., 2017), we will focus on the 280 participants in the mild cognitive impairment group for whom the complete information is available about the four demographic and clinical factors identified to be significantly associated with the AD conversion by Li et al. (2017). They are the participants' Alzheimer's Disease Assessment Scale Score of 13 items (ADAS13), the Rey auditory verbal learning test score of immediate recall (RAVLT.i), the functional assessment questionnaire score (FAQ) and the MRI volume of middle temporal gyrus (MidTemp). In addition to these four covariates, we will consider 162,194 SNPs, which were coded as 0,1 or 2 . Note that although there exists a much large number of SNPs in the data, most of them are constants for the subjects considered here and thus removed. Note that here most predictors are categorical and based on the simulation results given in Section 5, the proposed method may not perform well for the categorical covariates when the sample is small. On the other hand, also as seen in Section 5, it is expected to perform well for both categorical and continuous covariates for the given sample size here. 
Figure 4 presents the estimated dependence measures $\widehat{\omega}_{k}$ 's given by the model-free screening procedure proposed in the previous sections for the 162194 SNPs plus the four demographic and clinical factors, which correspond to $k=1, \ldots, 4$. It is apparent that as expected, the four demographic and clinical factors had higher correlations with the AD conversion time than individual SNPs. To determine the number of active SNPs, we should choose the 45 SNPs with the highest $\widehat{\omega}_{k}$ 's by using the selection rule $[n / \log (n)]$. To further see this, Figure 5 gives the largest $500 \widehat{\omega}_{k}$ 's from the largest to the smallest and Figure 6 displays the ratio sequence $\log \left(\widehat{\omega}_{(k)} / \widehat{\omega}_{(k+1)}\right)$ for the first 50 ratios. The former suggests that there may be at most 100 active covariates for the AD conversion time, and by using the proposed log-ratio criterion, the latter indicates that there may be around 10 or less than 20 active covariates.

\section{Discussion and Conclusion Remarks}

In this paper, we considered the variable selection or identifying of important or relevant variables for ultrahigh-dimensional IC failure time data and for the problem, a model-free screening procedure was proposed. To develop the proposed approach, a marginal utility $\omega_{k}$ was derived based on the DC between a redefined response or failure time variable and each 
covariate. One major advantage of the proposed screening method is that it does not involve any nonparametric estimation of a survival function or any complicated numerical optimization. Thus it can be easily implemented and the computation is fast. Furthermore, the procedure was shown to have the sure screening and ranking consistency properties and a datadriven log-ratio criterion was presented for the determination of the selection threshold value. The numerical results indicated that the proposed methodology works well for practical situations.

It should be noted that as most of SIS procedures, the proposed DCbased screening procedure is also a marginal approach developed based on marginal utilities, and one drawback of these marginal methods is that they may not perform well for the situations where covariates are jointly important but marginally unimportant. For the situation, one possible way is to generalize the proposed method to an iterative approach that can take the correlation among covariates into account or to develop a new screening method by directly incorporating the correlation information among covariates. Another common issue related to the feature screening of ultrahigh-dimensional data that has not discussed much in the literature is the analysis of the data after the feature screening or variable selection. Although many methods have been developed for either low-dimensional or 
high-dimensional data with respect to estimation or simultaneous variable selection and estimation, there seems to exist little literature on the joint analysis of the two steps or stages.

\section{Supplementary Materials}

Supplementary material includes the detailed proof of Theorems 1 and 2.

\section{Acknowledgements}

The authors wish to thank the Co-Editor, the Associate Editor and two reviewers for their many helpful and useful comments and suggestions that greatly improved the paper. Dr. Zhang and Dr. Liu's research was partially supported by the National Natural Science Foundation of China (NSFC 11901581, NSFC 11971362), Dr. Sun's research was partially supported by Washington University Institute of Clinical and Translational Sciences grant (\# CTSA 131)".

\section{References}

Fan, J., Feng, Y. and Song, R. (2011). Nonparametric independence screening in sparse ultrahigh-dimensional additive models. J. Am. Statist. Assoc. 106, 55-65.

Fan, J., Feng, Y. and Wu, Y. (2010). High-dimensional variable selection for Cox's proportion- 
ZHANG ET AL.

al hazards model. Borrowing Strength: Theory Powering Applications-a Festschrift for Lawrence D. Brown (pp. 70-86). Institute of Mathematical Statistics.

Fan, J. and Lv, J. (2008). Sure independence screening for ultrahigh dimensional feature space.

\section{J. R. Statist. Soc. B 70, 849-911.}

Fan, J., Samworth, R. and Wu, Y. (2009). Ultrahigh dimensional feature selection: Beyond the linear model. J. Mach. Learn. Res. 10, 2013-2038.

Fan, J. and Song, R. (2010). Sure independence screening in generalized linear models with NP-dimensionality. Ann. Statist. 38, 3567-3604.

Gorst-Rasmussen, A. and Scheike, T. (2013). Independent screening for single-index hazard rate models with ultrahigh dimensional features. J. R. Statist. Soc. B 75, 217-245.

Hu, Q., Zhu, L., Liu, Y., Sun, J. and Robison, L. L. (2020). Nonparametric screening and feature selection for ultrahigh-dimensional case II interval-censored failure time data. Biometrical J. 62, 1909-1925.

Huang, Q. and Zhu, Y. (2016). Model-free sure screening via maximum correlation. J. Mult. Anal. 148, 89-106.

Li, K., Chan, W., Doody, R. S., Quinn, J. and Luo, S. (2017). Prediction of conversion to Alzheimer's disease with longitudinal measures and time-to-event data. J. Alzheimer's Dis. 58, 361-371.

Li, R., Zhong, W. and Zhu, L. (2012). Feature screening via distance correlation learning. $J$. 


\section{FEATURE SCREENING FOR ULTRAHIGH-DIMENSIONAL IC DATA}

Am. Statist. Assoc. 107, 1129-1139.

Lin, Y., Liu, X. and Hao, M. (2018). Model-free feature screening for high-dimensional survival data. Sci. China Math. 61, 1617-1636.

Liu, Y., Zhang, J. and Zhao, X. (2018). A new nonparametric screening method for ultrahighdimensional survival data. Comput. Statist. Data Anal. 119, 74-85.

Scolas, S., El Ghouch, A., Legrand, C. and Oulhaj, A. (2016). Variable selection in a flexible parametric mixture cure model with interval-censored data. Stat. Med. 35, 1210-1225.

Song, R., Lu, W., Ma, S. and Jeng, X. J. (2014). Censored rank independence screening for high-dimensional survival data. Biometrika 101, 799-814.

Sun, J. (2006). The statistical analysis of interval-censored failure time data. Springer: New York.

Székely, G. J., Rizzo, M. L. and Bakirov, N. K. (2007). Measuring and testing dependence by correlation of distances. Ann. Statist. 35, 2769-2794.

Tibshirani, R. (2009). Univariate shrinkage in the Cox model for high dimensional data. Stat. Appl. Genet. Mol. 8, 1-18.

Turnbull, B. W. (1976) The empirical distribution with arbitrarily grouped, censored and truncated data. J. R. Statist. Soc. B 38, 290-295.

Wu, Y. and Cook, R. (2015). Penalized regression for interval-censored times of disease progression: selection of HLA markers in psoriatic arthritis. Biometrics $\mathbf{7 1}$, 782-791. 
Wu, Y. and Yin, G. (2015). Conditional quantile screening in ultrahigh-dimensional heterogeneous data. Biometrika 102, 65-76.

Zhao, H., Wu, Q., Li, G. and Sun, J. (2020). Simultaneous estimation and variable selection for interval-censored data with broken adaptive ridge regression. J. Am. Statist. Assoc. 115, 204-216.

Zhao, S. D. and Li, Y. (2012). Principled sure independence screening for Cox models with ultra-high-dimensional covariates. J. Mult. Anal. 105, 397-411.

Zhang, J., Liu, Y. and Cui, H. (2020). Model-free feature screening via distance correlation for ultrahigh dimensional survival data. Stat. Pap. https://doi.org/10.1007/s00362-02001210-3.

Zhang, J., Liu, Y. and Wu, Y. (2017). Correlation rank screening for ultrahigh-dimensional survival data. Comput. Statist. Data Anal. 108, 121-132.

Zhang, J., Yin, G., Liu, Y. and Wu, Y. (2018). Censored cumulative residual independent screening for ultrahigh-dimensional survival data. Lifetime Data Anal. 24, 273-292.

Zhou, T. and Zhu, L. (2017). Model-free feature screening for ultrahigh dimensional censored regression. Stat. Comput. 27, 947-961.

Zhu, L. P., Li, L., Li, R. and Zhu, L. X. (2011). Model-free feature screening for ultrahighdimensional data. J. Am. Statist. Assoc. 106, 1464-1475.

School of Statistics and Mathematics, Zhongnan University of Economics and Law, Wuhan, 
Hubei 430073, China

E-mail: jing66@zuel.edu.cn

Department of Mathematics and Statistics, The Hong Kong Polytechnic University, Hong Kong,

China

E-mail: dummoon@163.com

School of Mathematics and Statistics, Wuhan University, Wuhan, Hubei 430072, China

E-mail: liuyy@whu.edu.cn

Department of Statistics, University of Missouri, Columbia, Missouri 65211, U.S.A

E-mail: sunj@missouri.edu 
Table 1: The simulation results on $\mathcal{S}$ (the minimum model size needed to include all active covariates), $\mathcal{P}_{e}$ (the selection proportions for each active covariate) and $\mathcal{P}_{a}$ (the selection proportion for all active covariates) for set-up 1

\begin{tabular}{|c|c|c|c|c|c|c|c|c|c|c|}
\hline \multirow{3}{*}{$\frac{p}{2000}$} & \multirow[b]{2}{*}{$n$} & \multirow[b]{2}{*}{ Method } & \multirow[b]{2}{*}{ MMS } & \multirow[b]{2}{*}{ RSD } & \multicolumn{5}{|c|}{$\overline{\mathcal{P}_{e}}$} & \multirow[b]{2}{*}{$\mathcal{P}_{a}$} \\
\hline & & & & & $Z_{1}$ & $Z_{2}$ & $Z_{3}$ & $Z_{4}$ & $Z_{5}$ & \\
\hline & 100 & DC-SIS1 & 8.69 & 0.75 & 1.000 & 1.000 & 1.000 & 0.998 & 0.960 & 0.960 \\
\hline & & DC & 5.13 & 0.00 & 1.000 & 1.000 & 1.000 & 1.000 & 0.998 & 0.998 \\
\hline & 200 & DC- & 5.07 & 0.00 & 1.000 & 1.000 & 1.000 & 1.000 & 1.000 & 1.000 \\
\hline & & & 5.00 & 0.00 & 1.000 & 1.000 & 1.000 & 1.000 & 1.000 & 1.000 \\
\hline 4000 & 100 & DC- & 16.99 & 0.75 & 1.000 & 1.000 & 0.998 & 0.992 & 0.914 & 0.914 \\
\hline & & & 5.18 & 0.00 & 1.000 & 1.000 & 1.000 & 1.000 & 0.998 & 0.998 \\
\hline & 200 & $\mathrm{DC}$ & 5.04 & 0.1 & 1.000 & 1.000 & 1.000 & 1.000 & 1.000 & 1.000 \\
\hline & & DC-SIS & 5.00 & 0.00 & 1.000 & 1.000 & 1.000 & 1.000 & 1.000 & 1.000 \\
\hline
\end{tabular}

MMS: the average value of $\mathcal{S}$ among 500 replications; RSD: defined as $\mathrm{IQR} / 1.34$, where IQR denotes the interquartile range of $\mathcal{S}$ over 500 replications; DC-SIS1: another DC-based SIS procedure where we directly set $Y=\left(U, V, \Delta_{1}, \Delta_{2}, \Delta_{3}\right)$; DC-SIS: the proposed method in this paper. 
Table 2: The simulation results on $\mathcal{S}$ (the minimum model size needed to include all active covariates), $\mathcal{P}_{e}$ (the selection proportions for each active covariate) and $\mathcal{P}_{a}$ (the selection proportion for all active covariates) for set-up 2

\begin{tabular}{|c|c|c|c|c|c|c|c|c|c|}
\hline \multirow[b]{2}{*}{$p$} & \multirow[b]{2}{*}{$n$} & \multirow[b]{2}{*}{ Case } & \multirow[b]{2}{*}{ Method } & \multirow[b]{2}{*}{ MMS } & \multirow[b]{2}{*}{ RSD } & \multicolumn{3}{|c|}{$\mathcal{P}_{e}$} & \multirow[b]{2}{*}{$\mathcal{P}_{a}$} \\
\hline & & & & & & $Z_{1}$ & $Z_{5}$ & $Z_{10}$ & \\
\hline \multirow[t]{12}{*}{2000} & 100 & a) & DC-SIS1 & 35.33 & 11.19 & 0.734 & 0.986 & 0.998 & 0.726 \\
\hline & & & DC-SIS & 6.19 & 2.99 & 1.000 & 1.000 & 1.000 & 1.000 \\
\hline & & b) & DC-SIS1 & 853.55 & 1242.35 & 0.084 & 0.954 & 0.996 & 0.076 \\
\hline & & & DC-SIS & 30.90 & 8.21 & 0.788 & 0.998 & 1.000 & 0.786 \\
\hline & & c) & DC-SIS1 & 1491.33 & 434.70 & 0.010 & 0.004 & 0.012 & 0.000 \\
\hline & & & DC-SIS & 41.35 & 8.21 & 1.000 & 0.812 & 1.000 & 0.812 \\
\hline & 200 & a) & DC-SIS1 & 10.09 & 2.24 & 0.996 & 1.000 & 1.000 & 0.996 \\
\hline & & & DC-SIS & 5.32 & 2.24 & 1.000 & 1.000 & 1.000 & 1.000 \\
\hline & & b) & DC-SIS1 & 488.65 & 483.77 & 0.240 & 1.000 & 1.000 & 0.240 \\
\hline & & & DC-SIS & 10.07 & 2.24 & 0.996 & 1.000 & 1.000 & 0.996 \\
\hline & & c) & DC-SIS1 & 1520.49 & 429.29 & 0.022 & 0.010 & 0.022 & 0.000 \\
\hline & & & DC-SIS & 3.34 & 0.00 & 1.000 & 1.000 & 1.000 & 1.000 \\
\hline \multirow[t]{12}{*}{4000} & 100 & a) & DC-SIS1 & 47.58 & 14.18 & 0.716 & 0.980 & 0.988 & 0.698 \\
\hline & & & DC-SIS & 6.36 & 2.99 & 0.998 & 1.000 & 1.000 & 0.998 \\
\hline & & b) & DC-SIS1 & 1757.48 & 2524.81 & 0.052 & 0.922 & 0.998 & 0.052 \\
\hline & & & DC-SIS & 9.77 & 22.39 & 0.660 & 0.996 & 1.000 & 0.658 \\
\hline & & c) & DC-SIS1 & 3103.47 & 786.94 & 0.006 & 0.006 & 0.004 & 0.000 \\
\hline & & & DC-SIS & 83.49 & 27.05 & 0.988 & 0.690 & 1.000 & 0.682 \\
\hline & 200 & a) & DC-SIS1 & 10.51 & 2.24 & 0.988 & 1.000 & 1.000 & 0.988 \\
\hline & & & DC-SIS & 5.43 & 2.24 & 1.000 & 1.000 & 1.000 & 1.000 \\
\hline & & b) & DC-SIS1 & 979.00 & 1020.15 & 0.186 & 1.000 & 1.000 & 0.186 \\
\hline & & & DC-SIS & 10.05 & 1.68 & 0.998 & 1.000 & 1.000 & 0.998 \\
\hline & & c) & DC-SIS1 & 3049.69 & 728.54 & 0.008 & 0.004 & 0.006 & 0.000 \\
\hline & & & DC-SIS & 7.21 & 0.00 & 1.000 & 0.982 & 1.000 & 0.982 \\
\hline
\end{tabular}

case a): $\boldsymbol{Z} \sim N_{p}(\mathbf{0}, \boldsymbol{\Sigma})$; case b): $Z_{1}$ is generated from Bernoulli distribution with success probability 0.5 ; case $\mathrm{c})$ : all covariates $Z_{k}(k=1, \ldots, p)$ are generated from the Bernoulli distribution with success probability 0.5; MMS: the average value of $\mathcal{S}$ among 500 replications; RSD: defined as IQR/1.34, where IQR denotes the interquartile range of $\mathcal{S}$ over 500 replications; DCSIS1: the naive DC-based SIS procedure which set $Y=\left(U, V, \Delta_{1}, \Delta_{2}, \Delta_{3}\right)$; DC-SIS: the proposed method in this paper. 
Table 3: The simulation results on $\mathcal{S}$ (the minimum model size needed to include all active covariates), $\mathcal{P}_{e}$ (the selection proportions for each active covariate) and $\mathcal{P}_{a}$ (the selection proportion for all active covariates) for set-up 2

\begin{tabular}{ccccrrcccc}
\hline & & & & & & \multicolumn{4}{c}{$\mathcal{P}_{e}$} \\
\cline { 6 - 8 }$p$ & $n$ & Ratio & Method & MMS & RSD & $Z_{1}$ & $Z_{5}$ & $Z_{10}$ & $\mathcal{P}_{a}$ \\
\hline 2000 & 100 & I & DC-SIS1 & 1490.87 & 425.75 & 0.008 & 0.012 & 0.016 & 0.000 \\
& & & DC-SIS & 6.53 & 2.24 & 1.000 & 1.000 & 0.998 & 0.998 \\
& & II & DC-SIS1 & 1529.61 & 359.33 & 0.008 & 0.006 & 0.006 & 0.000 \\
& & DC-SIS & 8.67 & 2.24 & 1.000 & 1.000 & 0.978 & 0.978 \\
& & III & DC-SIS1 & 1508.07 & 424.07 & 0.008 & 0.010 & 0.012 & 0.000 \\
& & DC-SIS & 11.29 & 3.73 & 0.964 & 0.998 & 0.996 & 0.958 \\
& & & & & & & & \\
& \multirow{2}{*}{200} & I & DC-SIS1 & 1509.70 & 435.07 & 0.018 & 0.010 & 0.012 & 0.000 \\
& & DC-SIS & 5.22 & 1.49 & 1.000 & 1.000 & 1.000 & 1.000 \\
& \multirow{2}{*}{ II } & DC-SIS1 & 1516.77 & 413.43 & 0.014 & 0.028 & 0.022 & 0.000 \\
& & DC-SIS & 6.98 & 1.49 & 1.000 & 1.000 & 1.000 & 1.000 \\
& \multirow{2}{*}{ III } & DC-SIS1 & 1495.15 & 427.61 & 0.024 & 0.026 & 0.016 & 0.002 \\
& & DC-SIS & 8.24 & 2.99 & 1.000 & 1.000 & 1.000 & 1.000 \\
\hline
\end{tabular}

I: left-censored, right-censored, interval-censored rates of 20\%,20\%,60\%; II: left-censored, right-censored, interval-censored rates of $20 \%, 60 \%, 20 \%$; III: left-censored, right-censored, interval-censored rates of $60 \%, 20 \%, 20 \%$; MMS: the average value of $\mathcal{S}$ among 500 replications; RSD: defined as $\mathrm{IQR} / 1.34$, where IQR denotes the interquartile range of $\mathcal{S}$ over 500 replications; DC-SIS1: the naive DC-based SIS procedure which set $Y=$ $\left(U, V, \Delta_{1}, \Delta_{2}, \Delta_{3}\right)$; DC-SIS: the proposed method in this paper. 
Table 4: The simulation results on $\mathcal{S}$ (the minimum model size needed to include all active covariates), $\mathcal{P}_{e}$ (the selection proportions for each active covariate) and $\mathcal{P}_{a}$ (the selection proportion for all active covariates) for set-up 3

\begin{tabular}{|c|c|c|c|c|c|c|c|c|c|c|}
\hline \multirow[b]{2}{*}{$p$} & \multirow[b]{2}{*}{$n$} & \multirow[b]{2}{*}{$\mathrm{F}_{\epsilon}$} & \multirow[b]{2}{*}{ Method } & \multirow[b]{2}{*}{ MMS } & \multirow[b]{2}{*}{ RSD } & \multicolumn{4}{|c|}{$\mathcal{P}_{e}$} & \multirow[b]{2}{*}{$\mathcal{P}_{a}$} \\
\hline & & & & & & $Z_{1}$ & $Z_{2}$ & $Z_{9}$ & $Z_{10}$ & \\
\hline \multirow[t]{12}{*}{2000} & 100 & Norm & DC-SIS1 & 44.79 & 17.91 & 0.934 & 0.936 & 0.826 & 0.918 & 0.668 \\
\hline & & & DC-SIS & 5.45 & 0.75 & 0.998 & 0.998 & 0.992 & 0.996 & 0.984 \\
\hline & & Logistic & DC-SIS1 & 120.69 & 83.21 & 0.850 & 0.802 & 0.664 & 0.752 & 0.388 \\
\hline & & & DC-SIS & 16.97 & 5.97 & 0.984 & 0.980 & 0.914 & 0.974 & 0.858 \\
\hline & & Extreme & DC-SIS1 & 41.28 & 19.59 & 0.946 & 0.938 & 0.838 & 0.900 & 0.688 \\
\hline & & & DC-SIS & 7.42 & 0.75 & 1.000 & 0.994 & 0.976 & 0.994 & 0.966 \\
\hline & 200 & Norm & DC-SIS1 & 4.86 & 0.00 & 1.000 & 1.000 & 0.996 & 0.998 & 0.994 \\
\hline & & & DC-SIS & 4.01 & 0.00 & 1.000 & 1.000 & 0.998 & 1.000 & 0.998 \\
\hline & & Logistic & DC-SIS1 & 17.21 & 1.49 & 0.992 & 0.988 & 0.962 & 0.992 & 0.940 \\
\hline & & & DC-SIS & 4.26 & 0.00 & 1.000 & 1.000 & 0.998 & 1.000 & 0.998 \\
\hline & & Extreme & DC-SIS1 & 5.45 & 0.00 & 1.000 & 1.000 & 0.994 & 1.000 & 0.994 \\
\hline & & & DC-SIS & 4.02 & 0.00 & 1.000 & 1.000 & 1.000 & 1.000 & 1.000 \\
\hline \multirow[t]{12}{*}{4000} & 100 & Norm & DC-SIS1 & 83.56 & 41.04 & 0.910 & 0.890 & 0.794 & 0.870 & 0.568 \\
\hline & & & DC-SIS & 7.24 & 0.75 & 0.994 & 0.998 & 0.976 & 1.000 & 0.968 \\
\hline & & Logistic & DC-SIS1 & 227.90 & 142.91 & 0.766 & 0.706 & 0.612 & 0.704 & 0.266 \\
\hline & & & DC-SIS & 23.19 & 8.21 & 0.970 & 0.960 & 0.906 & 0.946 & 0.810 \\
\hline & & Extreme & DC-SIS1 & 92.87 & 39.18 & 0.890 & 0.870 & 0.820 & 0.872 & 0.586 \\
\hline & & & DC-SIS & 8.94 & 0.75 & 0.996 & 0.992 & 0.972 & 0.992 & 0.952 \\
\hline & 200 & Norm & DC-SIS1 & 5.99 & 0.00 & 0.998 & 0.998 & 0.998 & 1.000 & 0.994 \\
\hline & & & DC-SIS & 4.03 & 0.00 & 1.000 & 1.000 & 1.000 & 1.000 & 1.000 \\
\hline & & Logistic & DC-SIS1 & 18.52 & 3.73 & 0.996 & 0.982 & 0.938 & 0.988 & 0.904 \\
\hline & & & DC-SIS & 4.15 & 0.00 & 1.000 & 1.000 & 1.000 & 1.000 & 1.000 \\
\hline & & Extreme & DC-SIS1 & 5.29 & 0.00 & 1.000 & 0.998 & 0.998 & 0.998 & 0.994 \\
\hline & & & DC-SIS & 4.02 & 0.00 & 1.000 & 1.000 & 1.000 & 1.000 & 1.000 \\
\hline
\end{tabular}

$\mathrm{F}_{\epsilon}$ : the distribution of $\epsilon$; MMS: the average value of $\mathcal{S}$ among 500 replications; RSD: defined as IQR/1.34, where IQR denotes the interquartile range of $\mathcal{S}$ over 500 replications; DC-SIS1: the naive DC-based SIS procedure which set $Y=\left(U, V, \Delta_{1}, \Delta_{2}, \Delta_{3}\right)$; DC-SIS: the proposed method in this paper. 

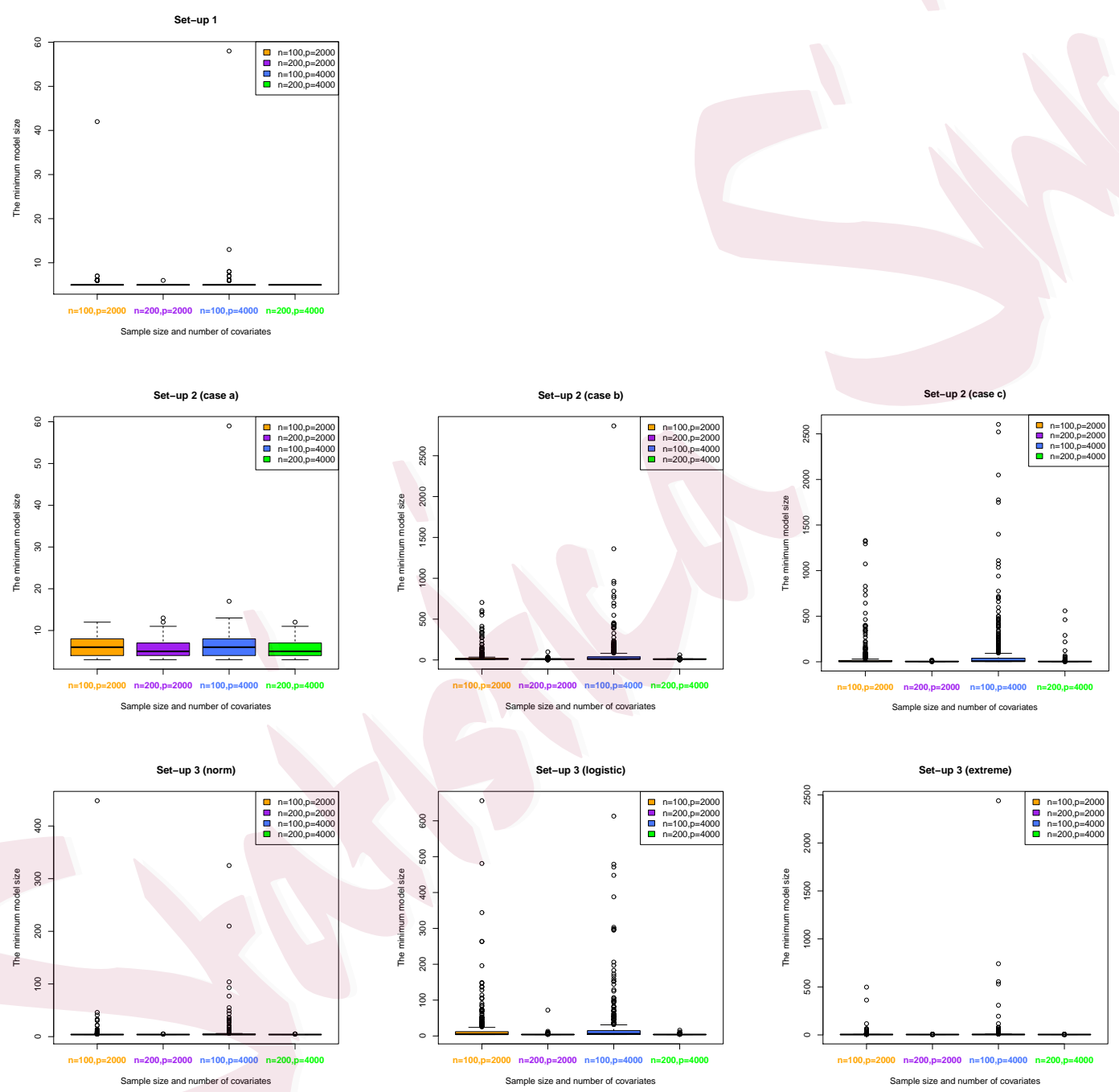

Figure 1: Box-plots of $\mathcal{S}$ for set-ups 1 (top), 2 (middle) and 3 (bottom). 


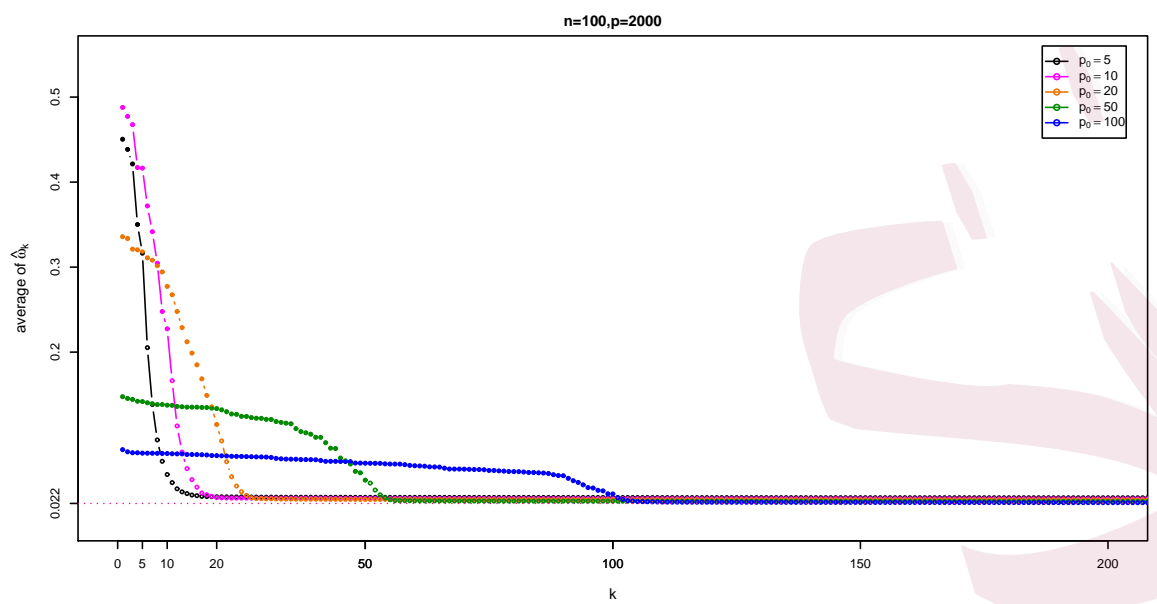

Figure 2: The plot of $\widehat{\omega}_{k}$ with the true model size $p_{0}=5,10,20,50,100$.

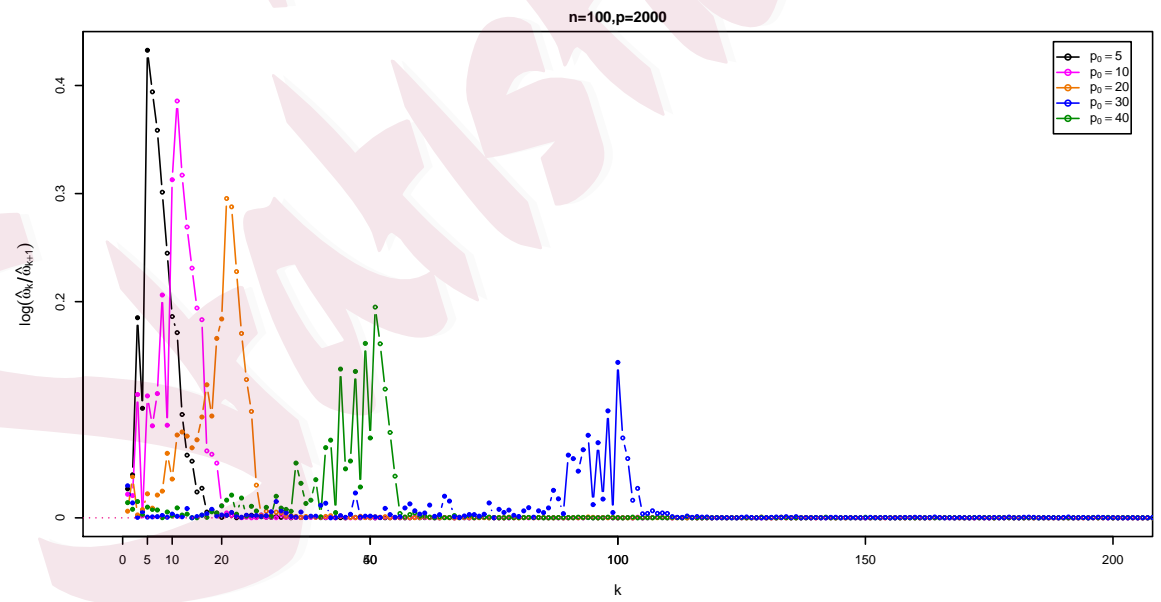

Figure 3: The plot of $\log \left(\widehat{\omega}_{(k)} / \widehat{\omega}_{(k+1)}\right)$ with the true model size $p_{0}=$ $5,10,20,50,100$. 


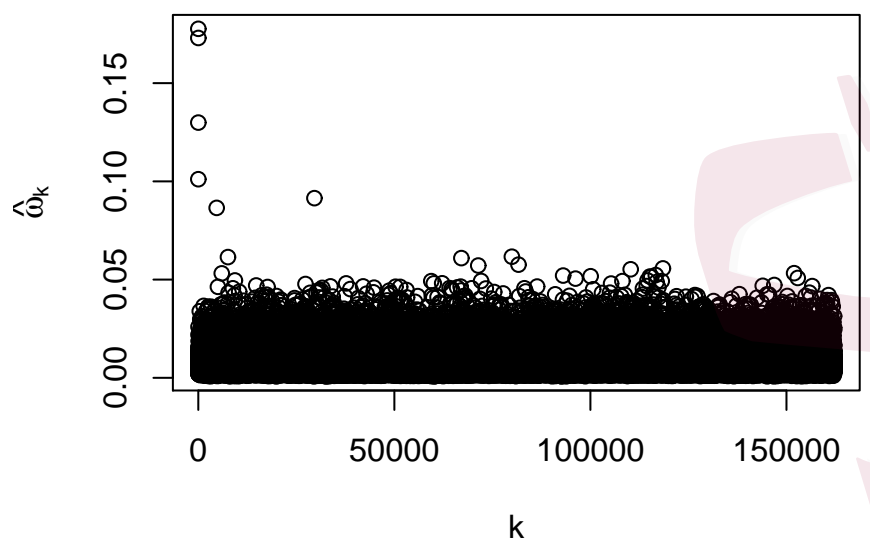

Figure 4: The $\widehat{\omega}_{k}$ values for all covariates for the AD example.

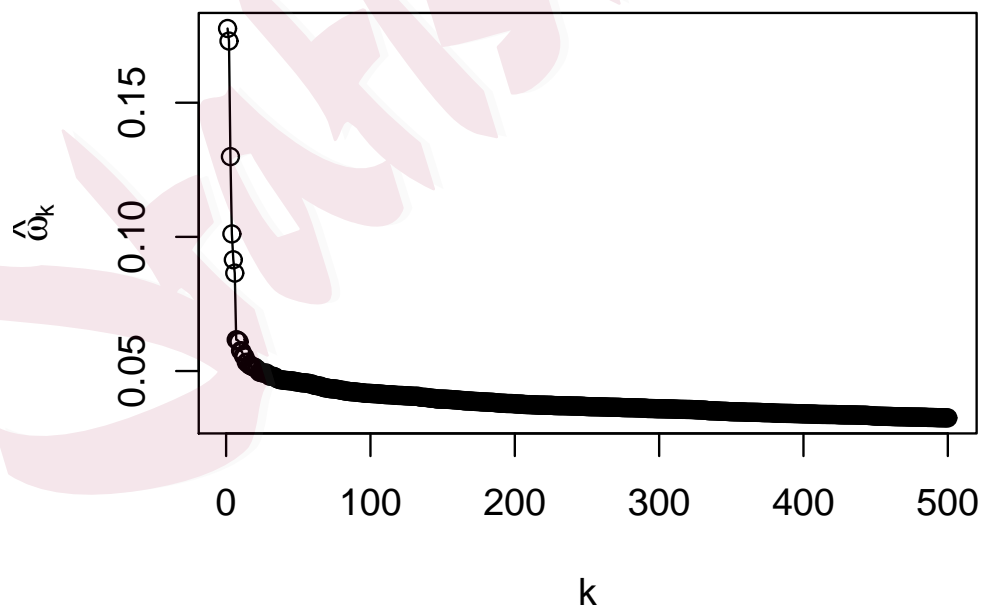

Figure 5: The 500 largest $\widehat{\omega}_{k}$ values for the AD example. 


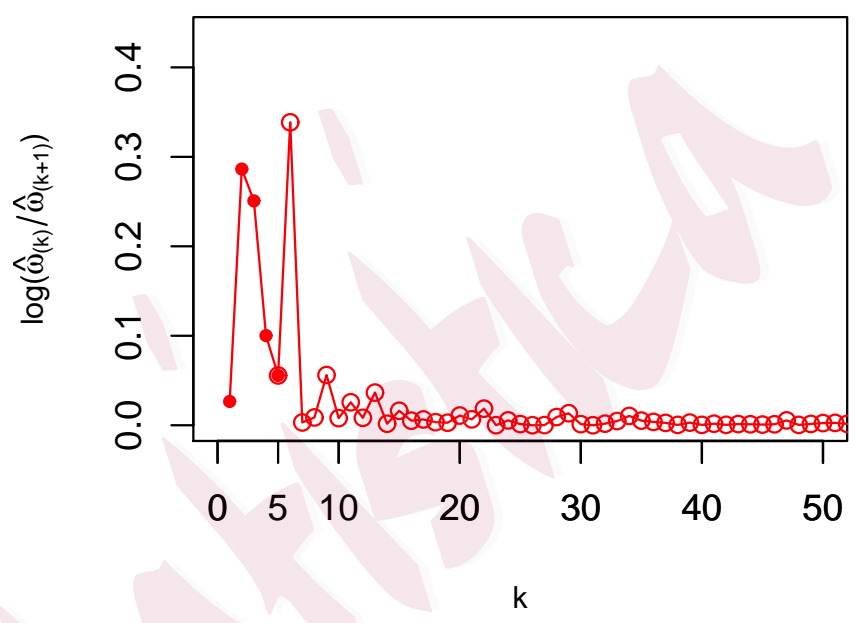

Figure 6: The scatter points of $\log \left(\widehat{\omega}_{(k)} / \widehat{\omega}_{(k+1)}\right)$ for the AD example. 